\title{
Metamaterial Inspired Electrically Small Multiband Monopole Antenna Using Single DNG MTM and Ring Resonators
}

Shiney Thankachan ( $\nabla$ shineyt156@gmail.com )

Cochin University of Science and Technology

Binu Paul

Cochin University of Science and Technology

\section{Research Article}

Keywords: WLAN frequencies, DNG structure, antenna.

Posted Date: July 13th, 2021

DOl: https://doi.org/10.21203/rs.3.rs-680615/v1

License: (c) (1) This work is licensed under a Creative Commons Attribution 4.0 International License.

Read Full License 


\title{
Metamaterial Inspired Electrically Small Multiband Monopole Antenna Using Single DNG MTM and Ring Resonators
}

\author{
${ }^{1 *}$ Shiney Thankachan, ${ }^{2}$ Binu Paul, \\ ${ }^{1 * 2}$ Division of Electronics Engineering, School of Engineering, \\ Cochin University of Science and Technology, Kochi-22. \\ $1^{*}$ Corresponding Author Email: shineyt156@gmail.com
}

\begin{abstract}
:
This paper proposes a new metamaterial inspired electrically small multi-band monopole antenna. The proposed antenna is capable of operating at DCS 1800 in the lower band. At the same time, in the higher band, it covers two wireless local area network (WLAN) bands at 2.4 and 5.2 GHz. This paper describes the design and detailed analysis of an electrically small (ka $=0.64<1$ ) antenna with a $-10 \mathrm{~dB}$ fractional bandwidth of $2.01 \%, 1.64 \%$ and $2.71 \%$ for tripleband operations with centre frequencies 1.80, 2.45 and 5.17 GHz. The compactness is achieved by the application of double negative metamaterial on a monopole antenna operating at $9 \mathrm{GHz}$. The proposed antenna has an overall compact electrical size $0.14 \lambda_{0} \times 0.14 \lambda_{0} \times 0.01 \lambda_{0}$ at 1.8 $\mathrm{GHz}$ and physical dimensions $24 \times 24 \times 1.6 \mathrm{~mm}^{3}$ including the ground. In this proposed ESA a second DNG structure is also incorporated to enhance its gain. This enables reliable operations at DCS 1800 and WLAN frequencies 2.4 and 5.2 GHz.
\end{abstract}

Keywords: WLAN frequencies, DNG structure, antenna. 


\section{INTRODUCTION}

The requirement for greater compactness of antenna in the field of wireless communication systems is increasing every day. Electrically small antennas (ESAs) attract researchers because they fulfil this requirement to a great extent despite their limitations such as lack of wide band and high gain. The distinct advantages of ESAs include compactness of size, simplicity of structure etc. and hence they can be incorporated into small sized devices quite easily. However because of their small electrical size, they have disadvantages like high reactance and low radiation resistance.

ESAs are compact antennas having physical dimensions far below one wavelength at their lowest frequencies of operation. One of the widely used mathematical definitions developed for ESA is $k a<1$ in which ' $k$ ' is the wave number $(2 \pi / \lambda)$ and ' $a$ ' is the spherical radius around the highest dimension of the unit cell. In ESAs decrease of the value of electrical size ' $k a$ ' closely correspond to the decrease of parameters like bandwidth, efficiency and gain. Radiation pattern of most of the ESAs are omnidirectional, similar to that of dipole antennas.

Different techniques such as employing a shorting post [1], microstrip fed slot techniques [2] and defected ground structure [3] have been proposed by researchers for developing ESAs for various applications. The performance of ESAs and larger antennas are more or less equal under matched conditions. However it is more difficult to achieve matching in ESAs when compared to larger antennas. In order to improve the impedance matching of ESAs two methods are commonly employed by researchers. In one method matching networks are used [4-6]. Here the entire system include the antenna and its matching network. Hence the entire system is cumbersome and deviates from the very concept of an ESA. A more preferable method of improving impedance matching is through structure modification of the antenna [79]. However there are certain difficulties in this method also. For example, even though modification of the structure of an ESA gives proper impedance matching with a $50 \Omega$ system 
connected to it, its Quality factor (Q) becomes very high and goes beyond the Chu limit. The bandwidth and the $\mathrm{Q}$ factor of an antenna are inversely proportional and hence this method also imposes a limitation on the antenna's bandwidth [10]. Secondly, impedance matching networks have some inherent losses associated with them. These losses may dominate over the radiation resistance of an ESA degrading its efficiency below the desired level. Thirdly, the limit for gain bandwidth proposed by Fano-Bode comes in as a limitation when we use lossless techniques of passive matching networks [11-13].

Antennas can also be made electrically small by using metamaterial (MTM) structures. The MTM structures help us in reducing the size of the device and obtaining various useful resonant frequency bands. Metamaterials are artificial materials that enable us to manipulate properties like permittivity and permeability in various frequency regions. MTMs are mainly made up of split ring resonator (SRR). MTMs have certain unique properties that enable them to improve the radiation properties of antennas, and achieve both structure compactness as well as desirable bandwidth. Metameterial can be used in different ways in ESA design. Metamaterial loaded, metamaterial inspired and metamaterial inspired with loading ESA designs are proposed by researchers.

The concept of MTM-inspired antennas was developed by Erentok and Ziolkowski [7]. MTM-inspired antenna is developed by placing an MTM unit-cell in the vicinity of the driven element, and utilise its features for enhancing the properties of the driven element. As a result the overall radiation efficiency and impedance matching of the antenna is improved without using additional matching networks [7-9]. The MTM unit-cell functions as the parasitic element in the near-field and becomes the dominant radiating component of the overall system at the desired resonant frequency [8].

MTM loading on monopole antennas function as additional capacitive and inductive elements in the near-field. However they reduce the bandwidth of the overall structure [14]. 
Electric-field-coupled and complementary electric-field-coupled resonators are parasitic structures used to load ESAs. But their bandwidth remain consistently below 2.55\% [15]. ACSfed dual band flipped MIMO antenna integrated in an actual USB module, which achieves fractional bandwidths upto $22 \%$ has been proposed [16]. Active devices can also be employed to improve the bandwidth of ESAs [17]. Antenna bandwidth can also be increased by using modified electric coupled resonator with the disadvantage of large size and low gain [18]. Yet another ESA which operates at $2.4 \mathrm{GHz}$ frequency is proposed [19]. It consists of a single parasitic MTM unit-cell attached to the ground plane, which reduces the size of the antenna considerably with good impedance matching. Moreover, in order to study the effect of NFRP element on structure of antenna, characteristic mode theory is also employed. Recently a very small antenna is designed using exclusively stamped metal for IoT/RFID applications with high radiation efficiency upto $80 \%$ and scalable to other operation bands 20 ].

In this paper, a compact triple-band MTM antenna design based on double negative metamaterial (DNG MTM) [20] and ring resonators is presented. Impedance matching and gain enhancement using alteration of ground plane and DNG loading are explored. An elaborate description and theoretical analysis of this proposed antenna are conducted and discussed in section 2, 3, 4 and 5 of this paper. In section 2 the evolution of the design of the antenna and geometrical parameters are described. Section 3 describes the extraction of DNG property in the MTM. Section 4 makes a parametric analysis of the various design parameters and identifies the optimum values for operating at the desired frequencies. In section 5 simulated and measured results are compared and analysed. Finally, in section 6 the advantages and novelty of the proposed antenna are described as the conclusion. 


\section{ANTENNA GEOMETRY AND DESIGN}

The triple-band MTM antenna structure proposed here is simulated and fabricated using low-cost FR-4 substrate with relative permittivity $\left(\varepsilon_{\mathrm{r}}\right) 4.4$, loss tangent $(\tan \delta) 0.02$ and substrate height (h) $1.6 \mathrm{~mm}$. The overall dimensions of the antenna are $24 \times 24 \times 1.6 \mathrm{~mm}^{3}$. The design of the proposed antenna is evolved through different stages in order to operate at all the desired frequency bands $1.8 / 2.4 / 5.2 \mathrm{GHz}$ by the optimization of various parameters. Figure 1 depicts the design stages.

The proposed design is evolved from a monopole operating at $9 \mathrm{GHz}$ with truncated ground (step 1 of Figure 1). In the next stage, this monopole is converted into metamaterial inspired monopole yielding two operating bands at $7 \mathrm{GHz}$ and $9 \mathrm{GHz}$ (step 2 of Figure 1)by incorporating a MTM structure exhibiting DNG property in C-band [21].

The MTM inspired monopole structure of step 2 is incorporated with two closed ring resonators with inner radius $\mathrm{R}_{2}$ and $\mathrm{R}_{3}$ respectively (Table 1 ), inorder to give two additional resonances at $4.5 \mathrm{GHz}$ and $5.5 \mathrm{GHz}$ as shown in step 3 and step 4 of Figure 1. Inclusion of two closed ring resonators increases the inductance effect and hence the ESA's capacitive reactance can be matched with this inductance. In step 5, ground structure is modified for giving proper impedance matching at lower bands and hence the overall structure resonate at the desired operating bands 1.8, 2.4 and 5.2 GHz. The corresponding input return loss $\left(\mathrm{S}_{11}\right)$ responses of the different stages of antenna evolution are plotted as Figure 2.

The configuration of the designed antenna is shown in Figure 3. Figures 3(a) and 3(b) show front view and back view of the final optimised structure of ESA operating at multifrequency bands. The final optimized parameters of the proposed antenna are given in Table.1. 


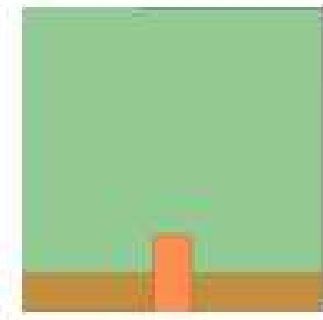

STEP 1

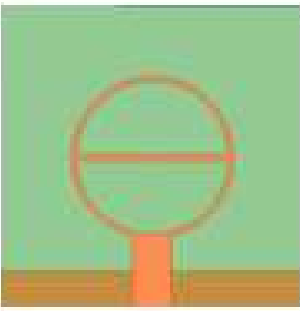

STEP 2

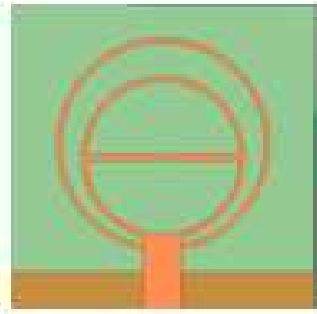

STEP 3

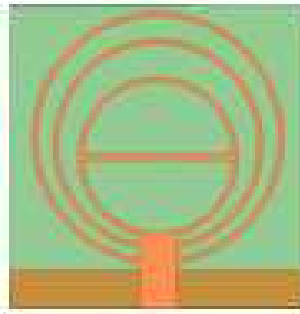

STEP 4

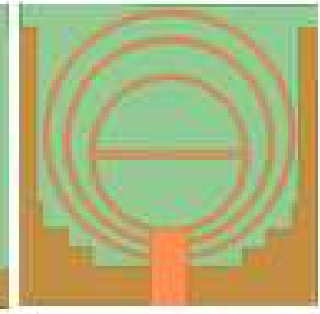

STEP 5

Figure 1. Antenna design evolution stages

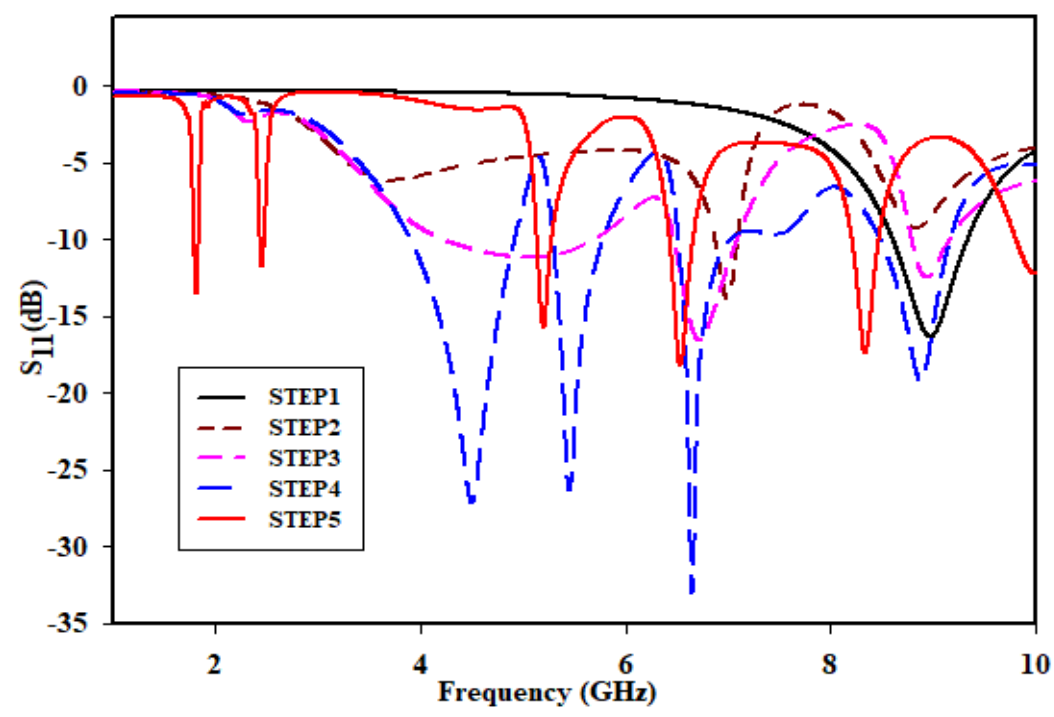

Figure 2. Return loss responses of various design evolution steps

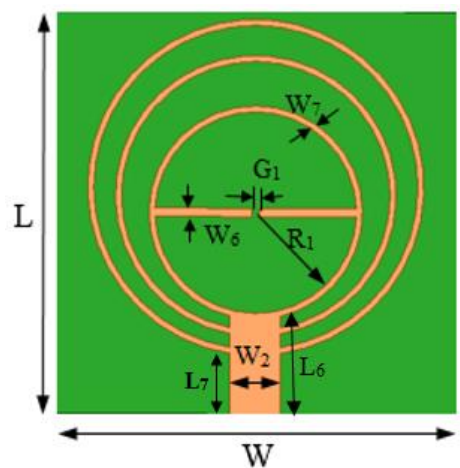

(a)

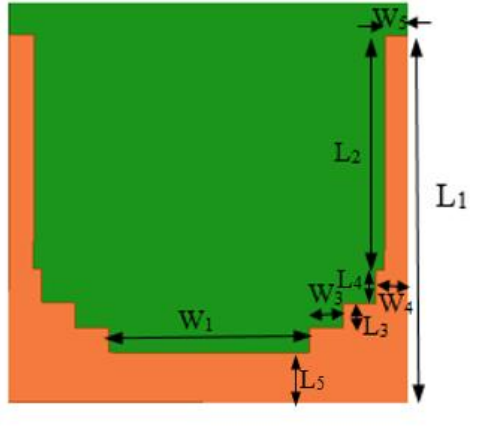

(b)

Figure 3. The proposed ESA (a) Front View (b) Back View

Table 1. Design parameters of the proposed ESA 


\begin{tabular}{|l|l|l|l|l|l|l|l|l|l|l|l|}
\hline Parameters & $\mathrm{L}$ & $\mathrm{L}_{1}$ & $\mathrm{~L}_{2}$ & $\mathrm{~L}_{3}$ & $\mathrm{~L}_{4}$ & $\mathrm{~L}_{5}$ & $\mathrm{~L}_{6}$ & $\mathrm{~L}_{7}$ & $\mathrm{~W}$ & $\mathrm{~W}_{1}$ & $\mathrm{~W}_{2}$ \\
\hline Size $(\mathrm{mm})$ & 24 & 22 & 14 & 1.5 & 2 & 3 & 6 & 3.6 & 24 & 12 & 3 \\
\hline Parameters & $\mathrm{W}_{3}$ & $\mathrm{~W}_{4}$ & $\mathrm{~W}_{5}$ & $\mathrm{~W}_{6}$ & $\mathrm{~W}_{7}$ & $\mathrm{G}_{1}$ & $\mathrm{R}_{1}$ & $\mathrm{R}_{2}$ & $\mathrm{R}_{3}$ & $\mathrm{R}_{4}$ & \\
\hline Size $(\mathrm{mm})$ & 2 & 2 & 1.5 & 0.5 & 0.35 & 0.2 & 6 & 8 & 10 & 7 & \\
\hline
\end{tabular}

\section{EXTRACTION OF THE DOUBLE NEGATIVE PROPERTY IN MTM}

The negative permittivity and negative permeability are retrieved from the S-parameters based on [21]. To extract the $S_{11}$ and $S_{21}$ characteristics of MTM, a two-port waveguide configuration as shown in Figure 4 can be used. For this boundary conditions are to be applied in such a way that the walls of the waveguide use a pair each, of both perfect magnetic conductor (PMC) and perfect electric conductor (PEC) along the planes $x-y$ and $y-z$ respectively. MTM structure is modelled as PEC on a dielectric slab located at the centre of the waveguide.

Two waveguide ports are placed parallel to x-z planes. Then the input wave is launched from port1 and $S_{11}$ and $S_{21}$ are determined. Using these values, permittivity, permeability, refractive index and impedance can be retrieved by S-parameter retrieval method using the following equations. These equations are implemented in MATLAB ${ }^{\mathrm{TM}}$.

$$
\begin{gathered}
Z= \pm \sqrt{\frac{\left(1+S_{11}\right)^{2}-S_{21}^{2}}{\left(1-S_{11}\right)^{2}-S_{21}^{2}}} \\
e^{i n k_{0} d}=\frac{S_{21}}{1-S_{11} \frac{Z-1}{Z+1}} \\
\left.n=\frac{1}{k_{0} d}\left\{\left[\ln \ln \left(e^{i n k_{0} d}\right)\right]^{\prime \prime}+2 m \pi\right]-i\left[\ln \ln \left(e^{i n k_{0} d}\right)\right]^{\prime}\right\}
\end{gathered}
$$


Where ' $k_{0}$ 'is the wavenumber in free space and ' $m$ ' represents branch index of refractive index which is an integer. Relative permittivity $\varepsilon$ and relative permeability $\mu$ can be calculated using the relations (4) and (5).

$$
\begin{aligned}
\varepsilon & =n / z \\
\mu & =n z
\end{aligned}
$$

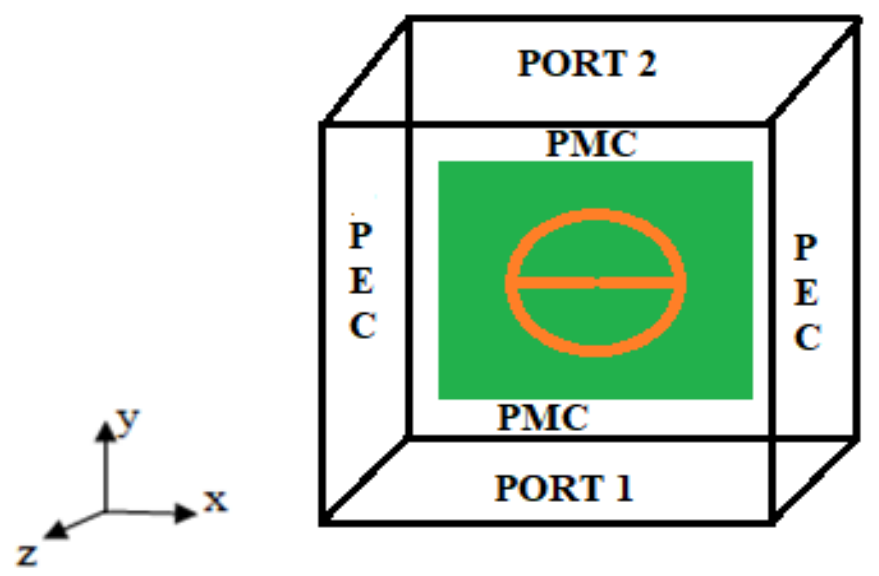

Figure 4. S-parameters retrieval waveguide setup

Figure 5 shows the extracted values of relative permittivity $\varepsilon$, relative permeability $\mu$, refractive index $n$ and impedance $z$. It can be observed in Figure 5 (a) that, both permittivity and permeability show negative values over a wide range in C-band. In Figure 5 (b) the DNG property is exhibited at where $n$ is negative. A passive material shows DNG behaviour when its $n$ shows negative values and $z$ shows positive values. 


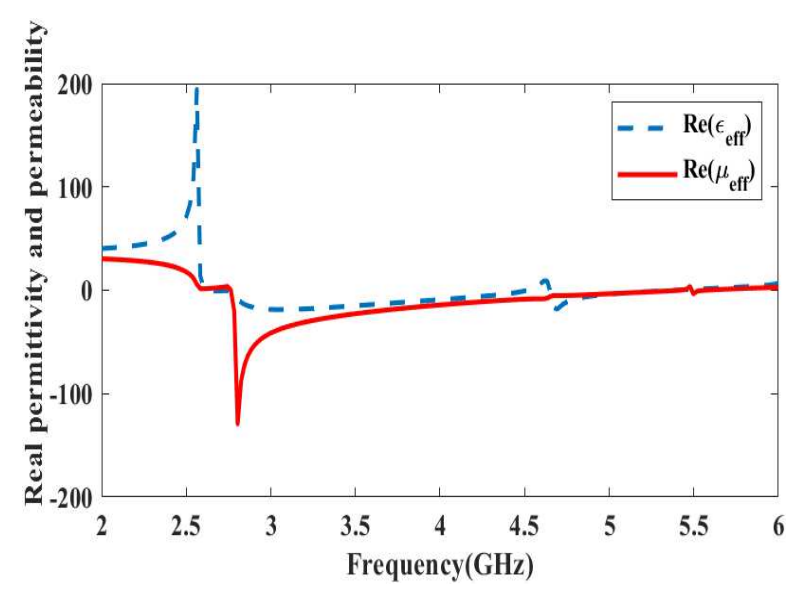

(a)

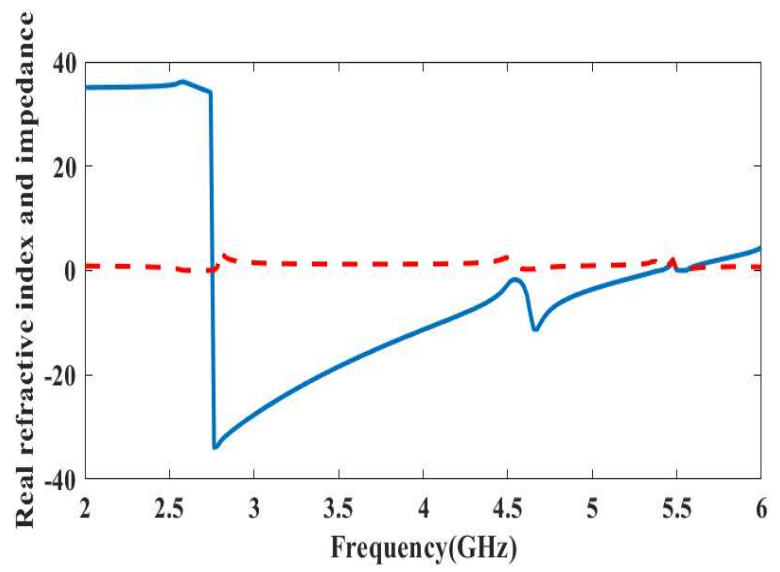

(b)

Figure 5. Retrieved results for (a) relative permittivity $\varepsilon$, relative permeability $\mu(b)$ refractive index $n$ and impedance $z$

\section{PARAMETRIC ANALYSIS}

Here, dependencies of various design parameters such as gap length, radius of DNG structure and radius of resonating rings on impedance matching, frequency of resonance and bandwidth are discussed. In general, the alteration of the length and width of ground affect only impedance matching leaving other parameters such as resonant frequency and bandwidth almost unaffected. Hence only affected parameters need to be optimized to obtain the required frequency bands 1.8/2.4/5.2 GHz. Therefore only parameters other than dimensions of ground are analysed in detail in this study.

\subsection{Effect of the Gap Length $\left(G_{1}\right)$}

Gap length $\left(\mathrm{G}_{1}\right)$ is one of the significant parameters because it sustains the DNG property of the metamaterial structure used in the proposed ESA. In figure 6(a) the impact of gap length on return loss $\left(\mathrm{S}_{11}\right)$ characteristics of the designed ESA is plotted. It can be found that the desired resonances remain unchanged as the $\mathrm{G}_{1}$ between the stubs of DNG is changed from 10 $\mathrm{mm}$ to $0.2 \mathrm{~mm}$. Yet, the optimum gap of $\mathrm{G}_{1}=0.2 \mathrm{~mm}$ is chosen because all the required frequency bands resonate with good matching and maximum gain at this gap length. 


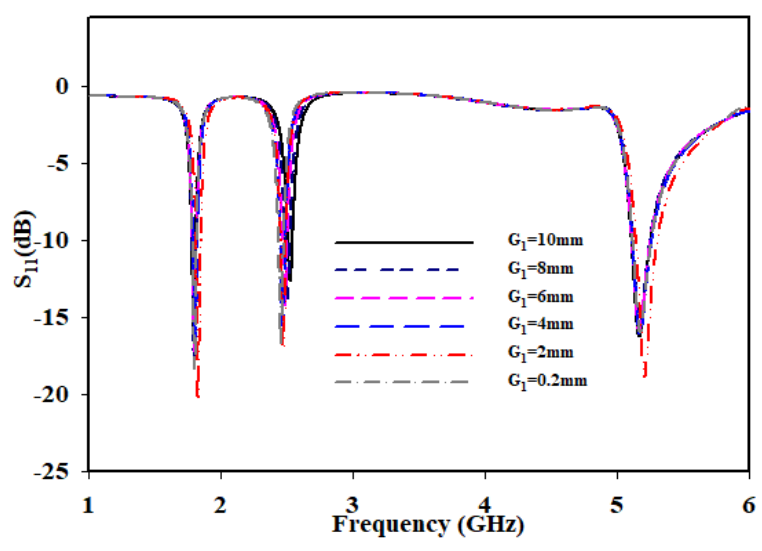

(a)

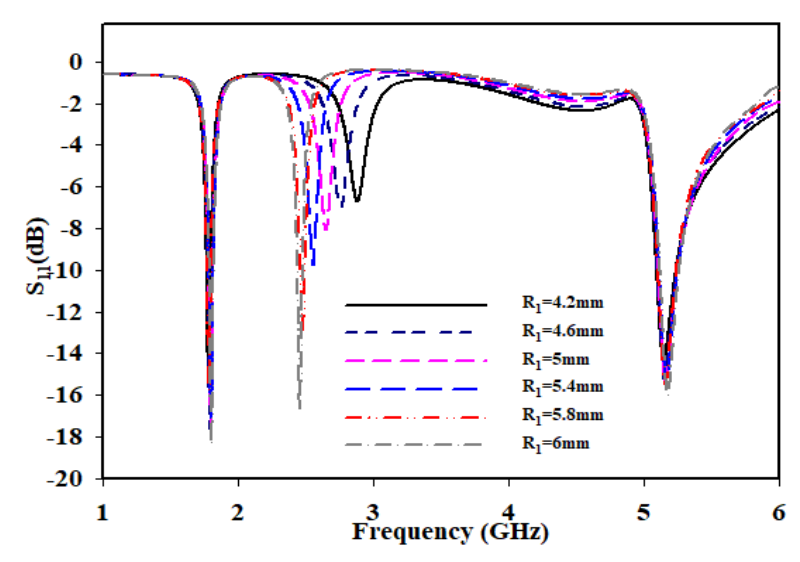

(b)

Figure 6. Effect of (a) gap length $G_{1}$ on $S_{11}$ (b) DNG radius $R_{1}$ on $S_{11}$

\subsection{Effect of DNG Radius $\left(\mathrm{R}_{1}\right)$}

The effect of DNG radius $\left(\mathrm{R}_{1}\right)$ on return loss is plotted in Figure 6(b). We can observe that the first and third resonances are unaffected as the DNG radius is altered. On the other hand, increase or decrease in the DNG radius has considerable impact on the second resonant frequency, which decreases as $\mathrm{R}_{1}$ increases.

\subsection{Effect of Radii of Resonating Rings $\left(\mathbf{R}_{2}, \mathbf{R}_{3}\right)$}

Figures $7(a)$ and (b) show the effect of $R_{2}$ and $R_{3}$ on resonance frequencies. $R_{2}$ significantly affects the second resonance whereas $R_{3}$ has its greatest effect on the first resonance. Alterations in both $\mathrm{R}_{2}$ and $\mathrm{R}_{3}$ cause minor shift in the third resonance also. 

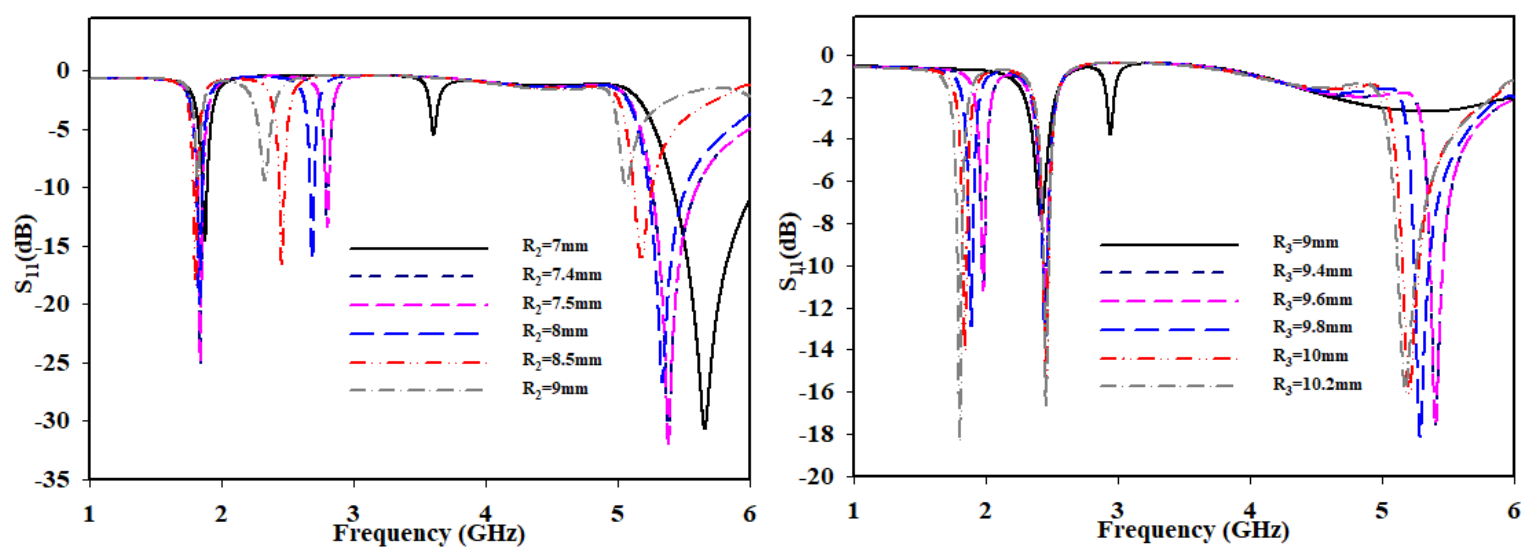

(a)

(b)

Figure 7. Effect of (a) $R_{2}$ on $S_{11}(b) R_{3}$ on $S_{11}$

\section{RESULTS AND DISCUSSIONS}

As per the values proposed in Table 1, a prototype has been fabricated and tested. The photographs of the fabricated antenna and the experimental setup for its measurement are shown in Figure 8. The simulations are done using Ansoft HFSS and measurements are made using Vector Network Analyzer. The simulated and measured $S_{11}$, plotted in Figure 9, are in good agreement. The $-10 \mathrm{~dB}$ impedance bandwidths at three operating bands $1.8 \mathrm{GHz}, 2.4 \mathrm{GHz}$ and $5.2 \mathrm{GHz}$ are $2.01 \%, 1.64 \%$ and $2.71 \%$ respectively, which cover the bands of DCS and some bands of WLAN simultaneously.

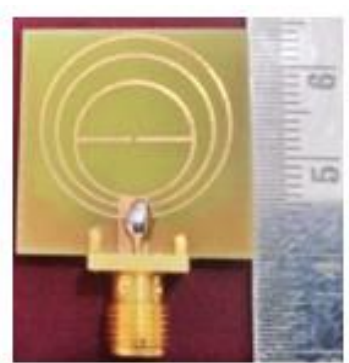

FRONT VIEW

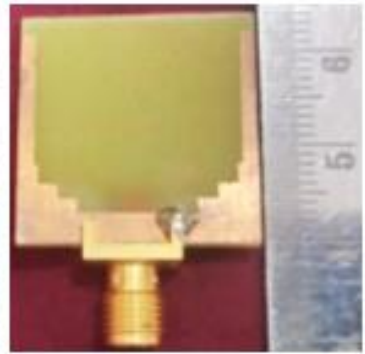

BACK VIEW

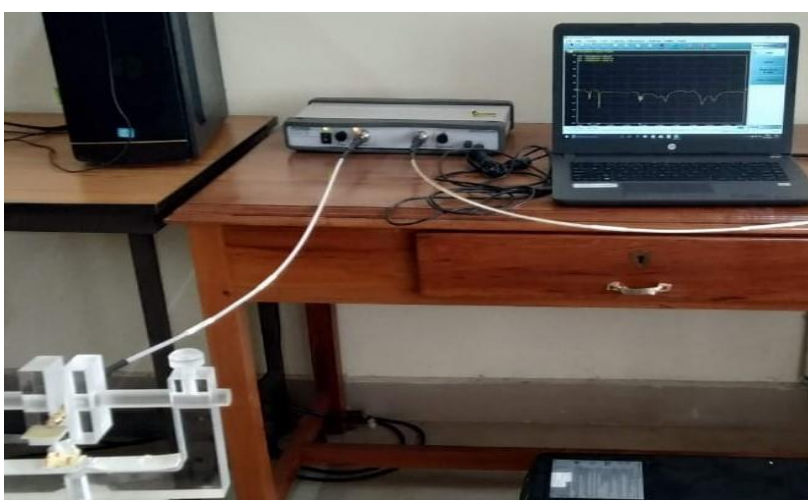

Figure 8. Photographs of the fabricated ESA and its measurement setup 


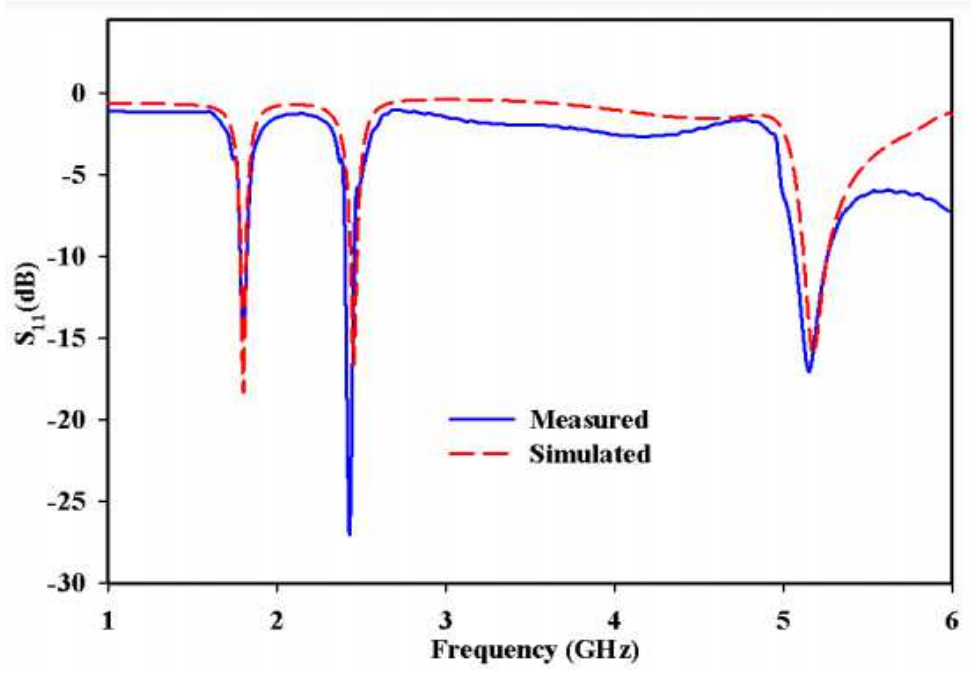

Figure 9. Simulated and measured $S_{11}$ of the proposed ESA.

To understand the influence of DNG structure on the monopole antenna, simulated surface current distribution at $1.8 \mathrm{GHz}, 2.4 \mathrm{GHz}$, and $5.2 \mathrm{GHz}$ frequencies are illustrated in Figure 10. The bands centered at 5.17 and $2.45 \mathrm{GHz}$ are caused by the DNG structure, as maximum surface current density is found in the DNG at these frequencies. The first band at $1.8 \mathrm{GHz}$ is caused by the presence of outer ring resonator, which contains maximum surface current density at this frequency. It also shows the coupling effect between ground plane and feed as well as coupling between DNG structure and second ring resonator. Coupling of the surface currents at the ground plane and feed also provide proper matching at first two bands.
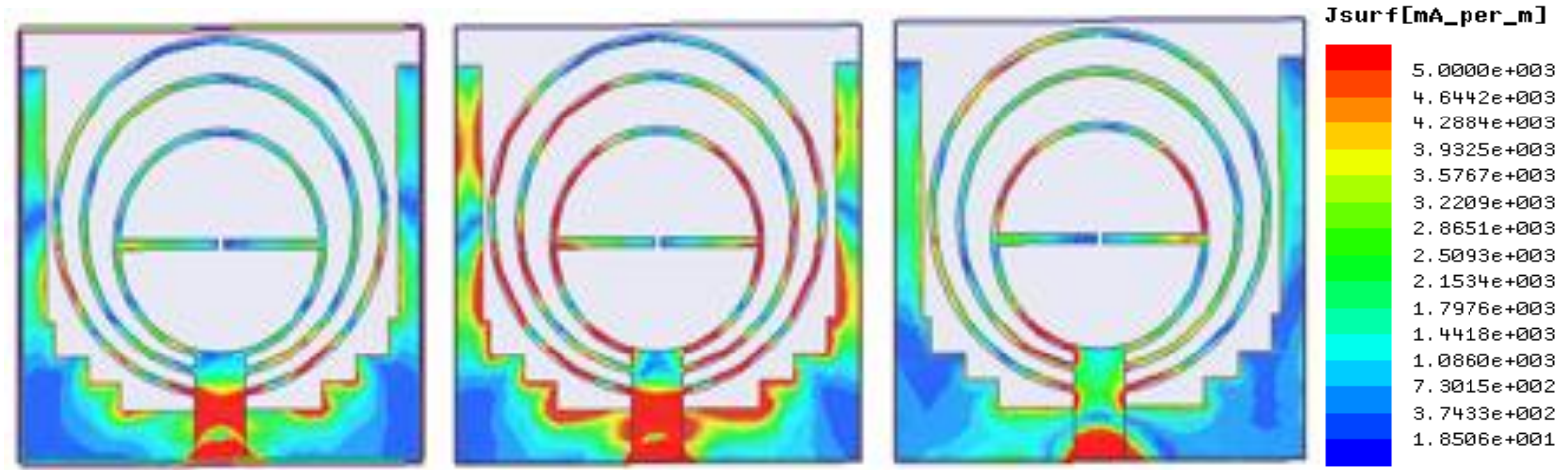

Figure 10. Current distributions at $1.8 \mathrm{GHz}, 2.4 \mathrm{GHz}$ and $5.2 \mathrm{GHz}$ 
The bandwidth of the proposed ESA is delimited by the theoretical limit for ESAs proposed by Chu [22]. This theoretical limit of quality factor $\left(Q_{\min }\right)$ for ESAs, is expressed by Chu as

$$
Q_{\min }=\frac{1}{k^{3} a^{3}}+\frac{1}{k a}
$$

where ' $k$ ' stands for the free space wave number, ' $a$ ' stands for the spherical radius around the highest dimension of the radiating part of the antenna.

The maximum bandwidth can also be expressed using Chu limit as

$$
F B W_{\max }=\frac{V S W R-1}{Q_{\min } \sqrt{V S W R}}
$$

Where $F B W_{\max }$ represents the maximum fractional bandwidth [23]. For the proposed ESA, $\mathrm{k}=$ $37.67 \mathrm{rad} / \mathrm{m}$ at the first resonant frequency $1.8 \mathrm{GHz}, \mathrm{a}=16.97 \mathrm{~mm}$ and therefore $\mathrm{ka}=0.64<$ 1. Hence, by definition, the proposed antenna is electrically small. On applying (6) and (7), the $F B W_{\max }$ that can be achieved at VSWR $=2$, is $13.15 \%$ and the bandwidth of $2.01 \%$ obtained is well below the maximum limit of bandwidth.

The $2 \mathrm{D}$ radiation patterns at $1.8 \mathrm{GHz}, 2.4 \mathrm{GHz}$, and $5.2 \mathrm{GHz}$ of the proposed ESA at the xz-plane and yz-plane are shown in Figure 11. The E and H-plane co-polarisation component of the proposed ESA exhibits radiation characteristics that are omnidirectional in all the frequency bands. In the E-plane and H-plane the antenna has low cross-polarisation component values for all the resonant frequencies, except the resonance at $1.8 \mathrm{GHz}$. 

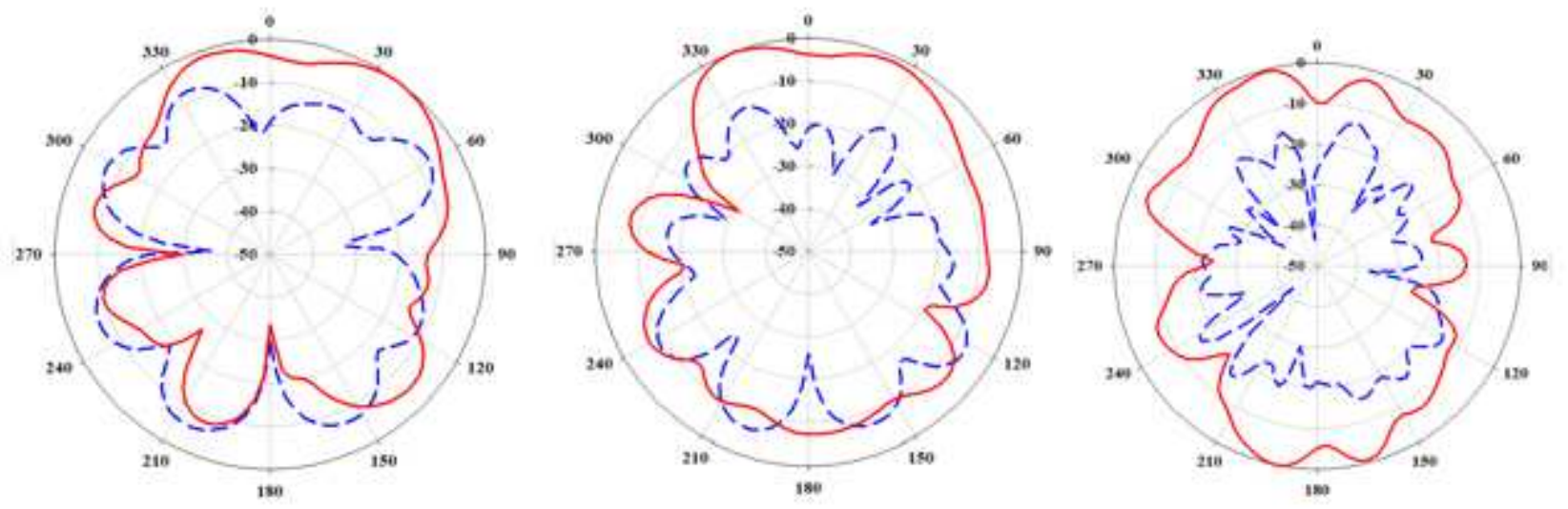

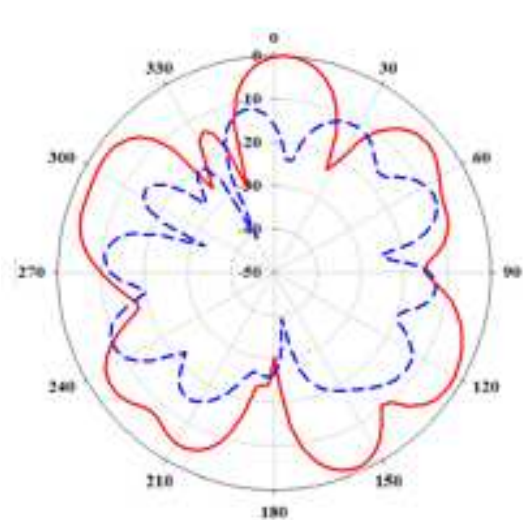

(a)

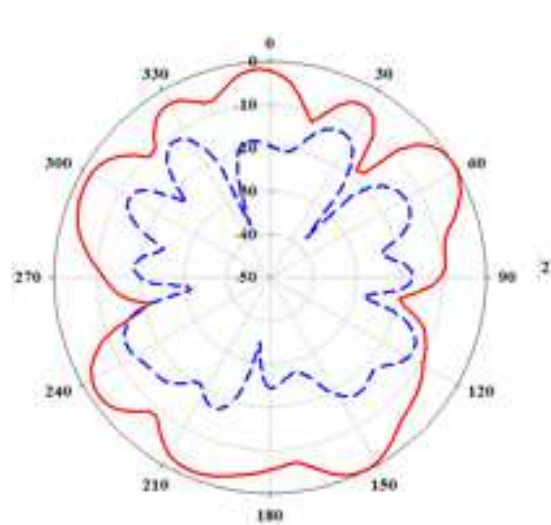

(b)

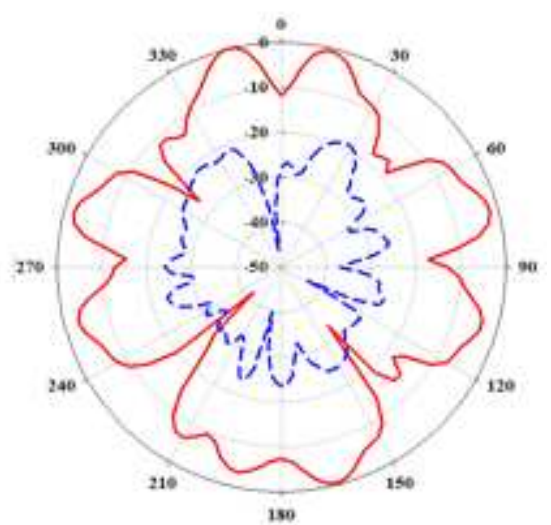

(c)

Figure 11. The proposed ESA's radiation patterns in $\mathrm{YZ}$ and $\mathrm{XZ}$ plane at (a) $1.8 \mathrm{GHz}$ (b) $2.4 \mathrm{GHz}$, (c) 5.2 GHz. (Blue: Co and Red: Cross polarization patterns).

It should be borne in mind that all the radiating energy of an ESA is found in its reactive nearfield, which seriously delimits its radiation efficiency. The reactive field can be converted into a propagating far-field by perturbing the near-field using periodic inclusions. Ziolkowski proposed a double negative metamaterial sphere for covering an ESA in order enhance its radiation efficiency [24]. The proposed antenna shows stable $S_{11}$ characteristics. However, the antenna shows peak gains of $-7.25 \mathrm{dBi}$ at $1.8 \mathrm{GHz},-1.8 \mathrm{dBi}$ at $2.4 \mathrm{GHz}$ and $0.12 \mathrm{dBi}$ at 5.2 GHz, respectively. This low gain can be improved using an additional DNG structure without altering return loss characteristics. Figure 12 shows the comparative evaluation of return loss characteristics of the proposed antenna and its modified design. The inductance of the 
additionally used DNG MTM can be used for matching ESA's high capacitive reactance. The modified structure is shown in Figure 13. Gain comparison of basic ESA with modified structure is shown in Figure 14. Peak gains obtained for the modified structure are $-5.6 \mathrm{dBi}$ at $1.8 \mathrm{GHz}, 2.9 \mathrm{dBi}$ at $2.4 \mathrm{GHz}$ and $1.7 \mathrm{dBi}$ at $5.2 \mathrm{GHz}$, respectively. Also the realisable gain of an ESA, is delimited by "Harrington bound" [25] which can be expressed using Equation (8)

$$
G=(k a)^{2}+2 k a
$$

The maximum feasible gain according to this equation is $1.68 \mathrm{dBi}$. The observed gain of the modified antenna design at $1.8 \mathrm{GHz}$ frequency is only $-5.6 \mathrm{dBi}$ which is well within the Harrington bound.

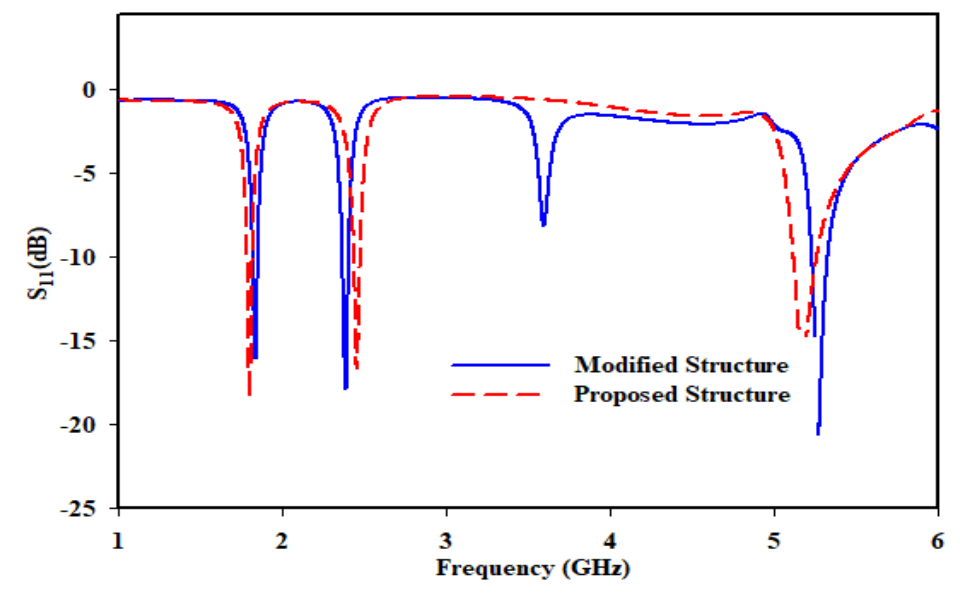

Figure 12. $\mathrm{S}_{11}$ comparison of the proposed antenna and its modified structure
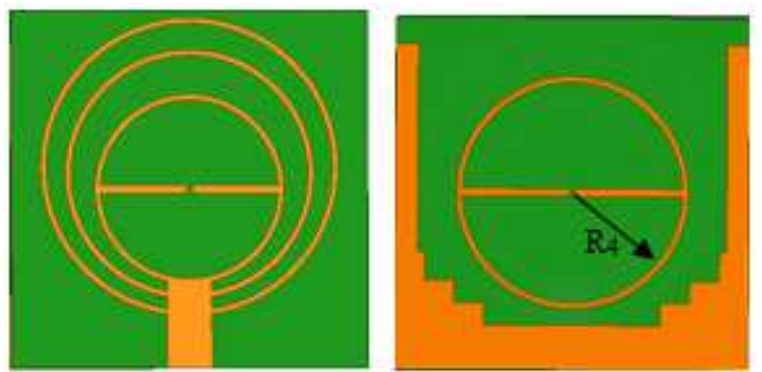

Figure 13. Modified Antenna Structure for gain enhancement (a) Front View (b) Back View 


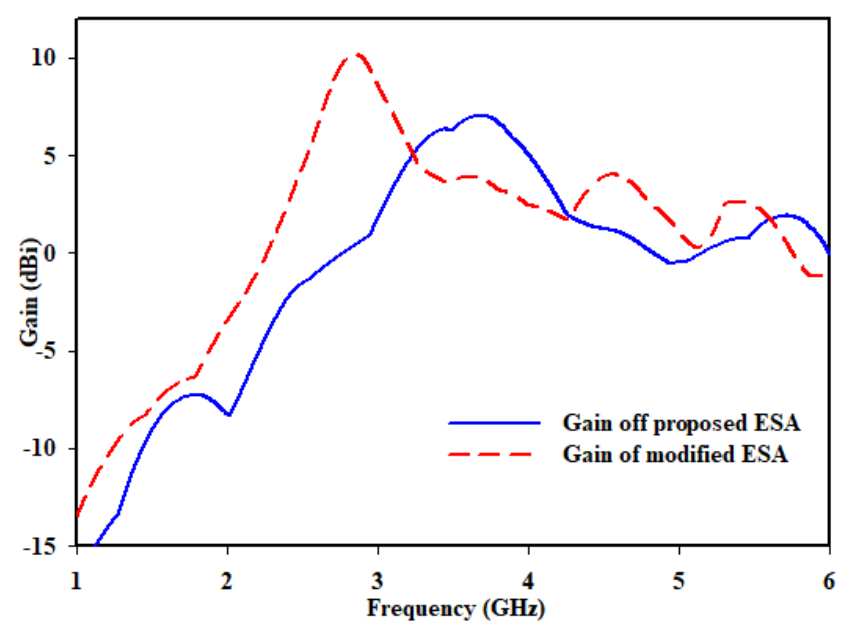

Figure 14. Gain comparison of the proposed ESA with its modified structure

\section{CONCLUSION}

This paper presents an electrically small metamaterial inspired multiband monopole antenna using single DNG MTM and ring resonators. The proposed ESA exhibits resonances at 1.8, 2.4 and $5.2 \mathrm{GHz}$ with an impedance bandwidth of $2.01 \%, 1.64 \%$ and $2.71 \%$ respectively which is under the maximum Chu limit of bandwidths. Additional DNG loading improves the gain within the Harrington bound at all resonant frequencies. The proposed antenna exhibits applicable radiation characteristics with one of the smallest electrical size of $0.14 \lambda_{0} \times 0.14 \lambda_{0}$ $\times 0.01 \lambda_{0}$ at $1.8 \mathrm{GHz}$. Moreover triple band operation is possible in the proposed ESA which is a distinct advantage over other ESAs which permit only single band or double band operation. Therefore the proposed ESA is found to be applicable in DCS 1800 and WLAN bands at 2.4 and $5.2 \mathrm{GHz}$.

\section{Funding}

Not applicable.

\section{Availability of data and materials}

All data generated or analysed in this study are included in this published article.

\section{Ethics approval and consent to participate}

Not applicable. 


\section{Consent for publication}

Not applicable.

\section{Competing interests}

The authors declare that they have no competing interests.

\section{Conflicts of Interest}

The authors declare no conflict of interest.

\section{REFERENCES}

[1] A. A. Salih and M. S. Sharawi, "A Dual-Band Highly Miniaturized Patch Antenna," IEEE Antennas and Wireless Propagation Letters, vol. 15, pp. 1783-1786, 2016, doi: 10.1109/LAWP.2016.2536678.

[2] L. Liu and B. Wang, "A Broadband and Electrically Small Planar Monopole Employing Metamaterial Transmission Line," IEEE Antennas and Wireless Propagation Letters, vol. 14, pp. 1018-1021, 2015, doi: 10.1109/LAWP.2015.2388762.

[3] Rikikumar Patel, Arpan Desai, and Trushit K. Upadhyaya, "An Electrically Small Antenna Using Defected Ground Structure for RFID, GPS and IEEE $802.11 \mathrm{a} / \mathrm{b} / \mathrm{g} / \mathrm{S}$ Applications," Progress In Electromagnetics Research Letters, Vol. 75, 75-81, 2018.

[4] Sussman-Fort S. E,"Matching network design using non-Foster impedances," Wiley. International Journal of RF and Microwave Computer-Aided Engineering, 16, (2), pp. 135$142,2006$.

[5] Sussman-Fort S. E. and Rudish R. M, "Non-Foster impedance matching of electrically-small antennas”, IEEE Transactions on Antennas and Propagation, 57, (8), pp. 2230-2241, 2009.

[6] Ruiyang Li, Gao Wei1, and Derek McNamara, "A Method for Matching Parasitic Unidirectional Electrically Small Array,” Progress In Electromagnetics Research Letters, Vol. 76, 121-126, 2018. 
[7] Erentok, A.and Ziolkowski, R. W, "Metamaterial-inspired efficient electrically small antennas," IEEE Transactions on Antennas and Propagation, 57, (3), pp. 691-707, 2008.

[8] Ziolkowski R. W., Jin, P. and Lin C.-C., "Metamaterial-inspired engineering of antennas," IEEE. Proc., 99, (10), pp. 1720-1731, 2011.

[9] Shih Y.-S. and Chi P.-L., "Miniaturized zeroth-order resonant antenna using couplingenhanced meandered structure,”(APSURSI), pp. 1378-1379, 2013.

[10] McLean J.S., "A Re-examination of the Fundamental Limits on the Radiation Q of Electrically Small Antennas," IEEE Transactions on Antennas and Propagation, vol.44, no.5, pp.672,, May 1996.

[11] R. M. Fano, "Theoretical Limitations on the Broadband Matching of Arbitrary Impedances," J. Franklin Inst., vol. 249, pp. 57-83, Jan. 1950, and pp. 139-155, Feb.1950.

[12] H. Bode, "Network Analysis and Feedback Amplifier Design," New York: Van Nostrand, 1947

p. 367.

[13] D. C. Youla, "A New Theory of Broadband Matching," IEEE Transactions on Circuit Theory, vol. CT-11, Mar. 1964.

[14] Antoniades, M. A.and Eleftheriades, G. V., "A broadband dual-mode monopole antenna using NRI-TL metamaterial loading," IEEE Antennas and Wireless Propagation Letters, 8, pp. 258-261, 2009.

[15] H. Odabasi, F. L. Teixeira, and D. O. Guney, "Electrically small, complementary electricfield-coupled resonator antennas,” J. Appl. Phys., vol. 113, no. 8, pp. 084903-1-4, Feb. 2013.

[16] Muhammad I. Magray1, Gulur S. Karthikeya, Khalid Muzaffar, and Shiban K. Koul,” Electrically Small ACS-Fed Flipped MIMO Antenna for USB Portable Applications,” Progress In Electromagnetics Research C, Vol. 95, 141-152, 2019. 
[17] M. Barbuto, A. Monti, F. Bilotti, and A. Toscano, "Design of a non-Foster actively loaded SRR and application in metamaterial-inspired components," IEEE Transactions on Antennas and Propagation, vol. 61, no. 3, pp.1219-1227, Mar. 2013.

[18] B. D. Bala, M. K. A. Rahim, and N. A. Murad, "Small electrical metamaterial antenna based on coupled electric field resonator with enhanced bandwidth,” Electron. Lett., vol. 50, no. 3, pp. 138-139, Jan. 2014.

[19] A. Sohrabi, H. Dashti, J. and Ahmadi-Shokouh, "Design and analysis of a Broadband electrically small antenna using characteristic mode theory", International Journal of Electronics and Communications (2019), doi: 10.1016/j.aeue.2019.152991

[20] Harry Contopanagos," An Electrically Small 3-D Folded Grounded Loop Antenna for Omnidirectional Connectivity," Progress In Electromagnetics Research C, Vol. 107, 245-258, 2021.

[21] Thankachan S, Paul B, Pradeep A and Moolat R. (2019), "Design and Characterisation of Simple Planar Metamaterial Structure with Double Negative Properties”, TENCON 2019 2019 IEEE Region 10 Conference (TENCON), 1231-1235.

[22] Chu LJ. "Physical limitations of omni-directional antennas". J Appl Physiol. 19:1163-1175., 1948.

[23] Fujimoto K. and Morishita H., "Fundamental limitations of small antennas", In Modern Small Antennas (pp. 23-38), Cambridge: Cambridge University Press, (2014). doi:10.1017/CBO9780511977602.005

[24] R. W. Ziolkowski and A. D. Kipple, "Application of double negative materials to increase the power radiated by electrically small antennas," in IEEE Transactions on Antennas and Propagation, vol. 51, no. 10, pp. 2626-2640, Oct. 2003, doi: 10.1109/TAP.2003.817561.

[25] Harrington RF, "Effect of antenna size on gain, bandwidth, and efficiency". J Res Natl Bur Stand D Radio Propag. 64D: 1-12, 1960. 